\title{
Decay rate of generalized solutions for equations of a viscous heat-conducting gas
}

\author{
Zhilei Liang ${ }^{1}$
}

${ }^{1}$ Southwestern University of Finance and Economics

June 5, 2021

\begin{abstract}
The large time behavior is considered for the solutions of the Navier-Stokes equations for one-dimensional viscous polytropic ideal gas in unbounded domains. Using the local anti-derivatives functions technique, we obtain the power type decay estimates for the generalized solutions as time goes to infinity
\end{abstract}

\section{Hosted file}

decay-rate-8.pdf available at https://authorea.com/users/418080/articles/524954-decay-rateof-generalized-solutions-for-equations-of-a-viscous-heat-conducting-gas 